\title{
Influence of Bi-Fe additive on properties of vanadium phosphate catalysts for $n$-butane oxidation to maleic anhydride
}

\begin{abstract}
The physico-chemical and catalytic properties of three ways of modified catalysts were studied, i.e. (i) the addition of both $\mathrm{Bi}$ and $\mathrm{Fe}$ (nitrate form) during the refluxing VOPO4.2H2O with isobutanol (Catalyst A), (ii) the simultaneous addition of BiFe oxide powder in the course of the synthesis of precursor VOHPO4. $0.5 \mathrm{H} 2 \mathrm{O}$ (Catalyst B) and (iii) the mechanochemical treatment of precursor VOHPO4.0.5H $2 \mathrm{O}$ and $\mathrm{BiFe}$ oxide in ethanol (Catalyst C). It was found that surface area of the modified catalysts has increased except Catalyst B. The reactivity of the oxygen species linked to V5+ and V4+ was studied by using H2-TPR, which also affected the catalytic performance of the catalyst. The conversion of nbutane decreases with an increment of oxygen species associated with V5+.
\end{abstract}

Keyword: $\quad$ Vanadium phosphate, n-Butane, Oxidation, Oxygen species, Maleic anhydride 